\title{
A PRECISE INEQUALITY OF DIFFERENTIAL POLYNOMIALS RELATED TO SMALL FUNCTIONS
}

\section{JUNFENG XU AND HoNGXUN Yi}

Abstract. In this paper, we consider the value distribution of the differential polynomials $\varphi f^{2} f^{\prime}-$ 1 where $f$ is a transcendental meromorphic function and $\varphi$ is a small function, and obtain a precise inequality by the reduced counting function.

Mathematics subject classification (2010): 30D35, 26D10. tribution.

Keywords and phrases: Meromorphic function, differential polynomials, Nevanlinna theory, value dis-

\section{REFERENCES}

[1] W. K. Hayman, Meromorphic functions, Clarendon Press, Oxford, 1964.

[2] I. LAINE, Nevanlinna theory and complex differential equations, Walter de Gruyter, Berlin-New York, 1993.

[3] E. Mues, Ueber ein problem von Hayman, Math. Z., 164, (1979), 239-259.

[4] J. F. XU, H. X. YI AND Z. L. ZhANG, Some inequalities of differential polynomials II, Mathematical Inequalities and Applications, 14, 1 (2011), 93-100.

[5] K. YAMANOI, The second main theorem for small functions and related problems, Acta Math., 192, (2004), 225-294.

[6] C. C. Yang And H. X. Yi, Uniqueness Theory of Meromorphic Functions, New York, Dordrecht, Boston, London, 2003.

[7] L. YANG, Value distribution theory, Springer, Berlin, Heidelberg, New York, 1993.

[8] Q. D. ZHANG, A growth theorem for meromorphic functions, J. Chengdu Inst. Meteor., 20, (1992), 12-20.

[9] Q. D. ZHANG, On the zeros of the differential polynomial $\varphi(z) f^{2}(z) f^{\prime}(z)-1$ of a transcendental meromorphic functions, J. Chengdu Inst. Meteor., 23, (1992), 9-17. 